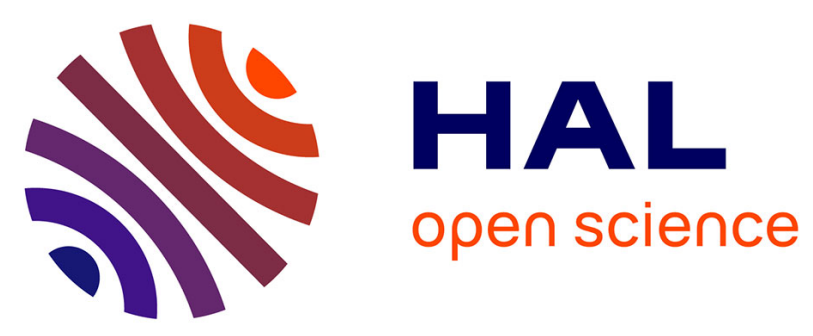

\title{
Monitoring unfractionated heparin therapy. 4 hour-stability of anti-Xa activity in unspun citrated tubes
}

Pierre Toulon, Anny Appert-Flory, Florence Fischer, Sylvain Buvat, Didier Jambou, Marie-Hélène Mahagne

\section{To cite this version:}

Pierre Toulon, Anny Appert-Flory, Florence Fischer, Sylvain Buvat, Didier Jambou, et al.. Monitoring unfractionated heparin therapy. 4 hour-stability of anti-Xa activity in unspun citrated tubes. Thrombosis Research, 2020, 186, pp.7 - 12. 10.1016/j.thromres.2019.10.019 . hal-03488557

\section{HAL Id: hal-03488557 https://hal.science/hal-03488557}

Submitted on 21 Dec 2021

HAL is a multi-disciplinary open access archive for the deposit and dissemination of scientific research documents, whether they are published or not. The documents may come from teaching and research institutions in France or abroad, or from public or private research centers.
L'archive ouverte pluridisciplinaire $\mathbf{H A L}$, est destinée au dépôt et à la diffusion de documents scientifiques de niveau recherche, publiés ou non, émanant des établissements d'enseignement et de recherche français ou étrangers, des laboratoires publics ou privés.

\section{다)(1) $(5$}

Distributed under a Creative Commons Attribution - NonCommerciall 4.0 International 
Monitoring unfractionated heparin therapy.

4 hour-stability of anti-Xa activity in unspun citrated tubes

\author{
Pierre Toulon ${ }^{1}$, Anny Appert-Flory ${ }^{1}$, Florence Fischer ${ }^{1}$, Sylvain Buvat ${ }^{1}$, \\ Didier Jambou ${ }^{1}$, and Marie-Hélène Mahagne ${ }^{2}$ \\ ${ }^{1}$ Université Côte d'Azur, CHU Nice, Hematology Department, Nice, France \\ 2 Université Côte d'Azur, CHU Nice, Neurology Department, Nice, France
}

* Address correspondence to: Dr Pierre Toulon

CHU Nice, Hôpital Pasteur

Service d'Hématologie Biologique

30 , avenue de la Voie Romaine

CS 51069

F-06001 Nice Cedex 1, France

Tel: + 33492038709

Fax: + 33492038595

E-mail: toulon.p@chu-nice.fr 
Key words: unfractionated heparin, monitoring, stability, anti-Xa activity, aPTT

Abbreviations: ACS: acute coronary syndrome

anti-Xa: anti-activated factor X (activity)

aPTT: activated partial thromboplastin time

AT: antithrombin

CAP: College of American Pathologists

$\mathrm{Cl}$ : confidence interval

CLSI: Clinical and Laboratory Standards Institute

CTAD: citrate, theophylline, adenosine and dipyridamole

CV: coefficient of variation

$\mathrm{F}$ : (clotting) factor

GFHT: French Working Group on Hemostasis and Thrombosis

ISO: International Organization for Standardization

IU: international unit

IV: intravenous

LMWH: low molecular weight heparin

NIBSC: National Institute for Biological Standards and Control

PF4: platelet factor 4

SC: sub-cutaneous

UFH: unfractionated heparin

VTE: venous thromboembolism

WHO: World Health Organization 


\section{Abstract}

Current guidelines recommend performing laboratory tests aimed at monitoring unfractionated heparin (UFH) treatments within a delay not exceeding 1 to 2 hour(s) after sampling when blood is collected into citrated tubes. As such a short delay could be an issue, we evaluated the potential impact of longer delays. For that purpose, two citrated tubes were obtained from patients on UFH: one was centrifuged and tested for anti-Xa activity and aPTT within $1 \mathrm{~h}$ after collection (T1h) and one was stored for $4 \mathrm{~h}$ at room temperature (T4h) before being processed. A total of 123 paired tubes were investigated. Anti-Xa activity was significantly lower at T4h than at $\mathrm{T} 1 \mathrm{~h}$, with a mean bias, calculated according to Bland-Altman, of $0.05 \mathrm{IU} / \mathrm{mL}$. Considering 0.30 to $0.70 \mathrm{IU} / \mathrm{mL}$ as the therapeutic range, there were 12 cases of discrepant test results (9.8\%). Most of them being around the lower limit of the therapeutic range had no impact on patients' management. APTT was significantly shortened $(p<0.0001)$ at T4h vs. T1h, with a mean bias of -8.1 sec. Considering antiXa correlated aPTT therapeutic range, 29 cases of discrepant test results (23.6\%) were found, $10 \%$ would have induce dosage changes. The concordance between anti-Xa activities measured at T4h and T1h was excellent (kappa=0.813) and good for aPTT (kappa=0.661). In conclusion, extending the delay between blood collection and measurement of tests prescribed for monitoring UFH therapy up to $4 \mathrm{~h}$ was found to lead to a systematic reduction in both anti-Xa activity and aPTT in unspun citrated tubes. As changes at T4h were limited and had few clinically relevance than the ones observed with aPTT testing, a 4h-delay was found to be acceptable for anti-Xa activity. The maximum delay for aPTT should remain around $1-2 \mathrm{~h}$ as changes were more relevant. 


\section{Introduction}

Unfractionated heparin (UFH), a sulfated polysaccharide extracted from porcine intestinal mucosa, enhances the inhibitory activity of antithrombin (AT), a natural anticoagulant that inhibits most activated clotting factors $(F)$, particularly FXa and FIla $[1,2]$. UFH has been in clinical use for more than half a century, and despite the growing interest for low molecular weight heparin derivatives (LMWH) and direct oral anticoagulants, it is still widely used for different indications including the treatment of acute thrombosis such as venous thromboembolism (VTE), acute coronary syndromes (ACS), and other thrombotic diseases [3]. UFH is administered parenterally using either intravenous (IV) or sub-cutaneous (SC) route. The therapeutic window of UFH is narrow with a risk of thrombosis in case of under-dosing and a risk of bleeding in case of over-dosing [4]. Altogether with the highly variable between-individuals anticoagulant response to UFH [5], this implies that a careful laboratory monitoring of UFH treatments is mandatory [3]. For that purpose, there are two main options i) to measure the heparin-enhanced inhibitory activity of AT toward purified activated factors such as FXa or Flla, usually using chromogenic substrate-based assays or ii) to evaluate the prolongation of the activated partial thromboplastin time (aPTT), a global clotting assay that reflects the ability of heparin to enhance the inhibitory activity of AT against Flla, FXa, and other activated clotting factors $[3,6]$. However, ensuring a proper monitoring of UFH treatments has to overcome one main critical issue, as UFH present in the patient's blood could be potentially neutralized in vitro by platelet factor 4 (PF4) released from platelet a-granules after their activation during or after venipuncture [7], leading to lowered anti-activated factor $\mathrm{X}$ (anti-Xa) activity and shortened aPTT test results. To circumvent that issue, it is recommended performing tests aimed at evaluating the anticoagulant activity of UFH as soon as possible after blood collection. Actually, and despite scarce evidence, the current Clinical and Laboratory Standards Institute (CLSI) guideline recommends a maximum delay of 1 hour between blood sampling and testing for anti-Xa activity or aPTT prescribed for monitoring UFH therapy, when blood is collected into citrated tubes [8]. Such a short delay was also recommended by the French Working Group on Hemostasis and 
Thrombosis (GFHT) in its latest guidelines, even though a 2 hour-delay was found to be acceptable for unspun tubes [9].

As the economic pressure led to a consolidation of clinical laboratories into so-called "core labs", during the last years, the delay between specimen collection and its delivery to the production site has dramatically increase due to longer distances between these two sites. In such conditions, a 1 hour-delay should be a limitation in some cases, making these recommendations difficult to apply in routine practice. 


\section{Aim and design of the study}

As such a short delay between 1 and 2 hours could raise logistical issues, particularly at multisite centres, the present study was carried out in order to evaluate the potential impact of a longer delay on the results of test aimed at monitoring UFH therapy when blood was collected into citrated tubes. For that purpose, 2 evacuated citrated tubes were collected from patients on UFH: one was centrifuged and tested within $1 \mathrm{~h}$ after blood collection (T1h) and one was stored unspun for $4 \mathrm{~h}$ at room temperature (in the range between $+18^{\circ} \mathrm{C}$ and $+25^{\circ} \mathrm{C}$ ) before being centrifuged and immediately analyzed (T4h). 


\section{Patients, materials and methods}

\section{Evaluated samples}

Venous blood was collected into polymer evacuated tubes containing $0.109 \mathrm{M}$ sodium citrate (1 vol./9 vol.) (Vacutainer, Becton-Dickinson, Le-Pont-de-Claix, France), according to international recommendations [10]. Blood samples were routinely handled according to the current recommendations for the preanalytical phase [11,12]. Accordingly, plasma was obtained by centrifugation at $2,200 \times g$ and $+18^{\circ} \mathrm{C}$ for 15 minutes $(\mathrm{min})$, and the delay between centrifugation and analysis was below $10 \mathrm{~min}$.

\section{Patients}

During the 2 month-duration of the study, 138 samples were received in a single accredited laboratory. Incomplete doublets of tubes $(n=2)$, underfilled tubes $(n=2)$ or hemolyzed samples $(n=1)$ were excluded from the study. Similarly, test results with a baseline anti-Xa activity below the lower limit of quantitation of the technique $(n=7)$ or above the upper limit of the calibration curve $(n=1)$ were not included in the statistical analysis, and the same applied to samples with aPTT at T0 above 150 sec. ( $n=3$, including the sample with a high anti-Xa activity). Finally, we evaluated one hundred and twenty-three samples obtained from 56 patients. There were 34 males and 22 females, with a mean age of 66.5 years (range: 19 - 96). Indication of UFH therapy was a recent ischemic stroke in all cases. Even though the standard treatment in the acute phase of stroke is aspirin and low molecular weight heparin in preventive doses, unfractionated heparin was used in patients considered to be at high embolic risk, particularly valvular or non-valvular atrial fibrillation, who had extensive cerebral ischemia and therefore risk of hemorrhagic transformation. The choice of UFH was made in these cases as a precautionary measure to closely monitor the anticoagulation and to keep the possibility of stopping in the event of hemorrhagic transformation. None was treated with any antiplatelet agents e.g. aspirin at the time of the blood sampling. In addition, it could be mentioned that none of the patients included in this study had renal failure. Moreover, IV UFH has never been used in the acute phase of stroke, particularly after thrombolysis 
$(n=8)$. UFH was administered using the IV route in all patients, but one in whom the SC route was used, with a mean daily UFH dosage of 21.000 international units (IU) (range: 13.500 - 42.000). The study was carried out in accordance with The Code of Ethics of the World Medical Association (Declaration of Helsinki) for experiments involving humans, after being approved by the local Ethics Committee. All patients gave their informed consent to participate in the study.

\section{Materials and methods}

Anti-Xa activity, expressed in IU/mL, was evaluated using a commercially available chromogenic substrate-based assay (Biophen Heparin LRT, Hyphen Biomed, Neuville-sur-Oise, France). The calibration curve was performed using a set of calibration plasmas (Biophen Heparin Calibrator) from the reagent manufacturer (Hyphen Biomed) traceable to the National Institute for Biological Standards and Control (NIBSC) / World Health Organization (WHO) $3^{\text {rd }}$ international standard for LMWH. The upper limit of the calibration curve was $1.32 \mathrm{IU} / \mathrm{mL}$ using the specific lot of reagent used, and the lower limit of quantitation was $0.10 \mathrm{IU} / \mathrm{mL}$. The within-run precision of the technique, evaluated as the coefficient of variation (CV, in \%) using lyophilized plasma samples, was in the range from $8.6 \%$ (for a mean anti-Xa activity $=0.17 \mathrm{IU} / \mathrm{mL}$ ) to $2.8 \%$ (for a mean anti-Xa activity=0.45 $\mathrm{IU} / \mathrm{mL}$ ) whereas the between-run CV was in the range from $8.6 \%$ (for a mean anti-Xa activity=0.20 $\mathrm{IU} / \mathrm{mL}$ ) to $2.1 \%$ (for a mean anti-Xa activity=0.44 $\mathrm{IU} / \mathrm{mL}$ ), as mentioned in the reagent package insert (version 01-2017). The locally defined imprecision of the technique was $16 \%$ for anti-Xa activities around $0.50 \mathrm{IU} / \mathrm{mL}$.

In order to confirm our results, we evaluated in a subset of samples $(n=62)$ another chromogenic assay (HemosIL Liquid Heparin, Instrumentation Laboratory, IL, Bedford, MA, USA) that was calibrated using a dedicated lyophilized set of calibration plasmas (HemosIL Calibrators, IL) traceable to the NIBSC / WHO international standards for UFH $\left(6^{\text {th }}\right)$ and LMWH $\left(3^{\text {rd }}\right)$. The upper limit of the calibration curve was $2.0 \mathrm{IU} / \mathrm{mL}$ using the specific lot of reagents used and the lower limit of quantitation was $0.10 \mathrm{IU} / \mathrm{mL}$. The within-run precision of the technique, evaluated as the CV using lyophilized plasma samples, was in the range from $1.9 \%$ (for a mean anti-Xa activity=0.33 
$\mathrm{IU} / \mathrm{mL}$ ) to $1.3 \%$ (for a mean anti-Xa activity=0.63 IU/mL) whereas the total $\mathrm{CV}$ was in the range from $4.0 \%$ to $2.0 \%$ respectively, as mentioned in the reagent package insert (version 06/2017).

The usual anti-Xa activity therapeutic range for UFH treatments was between 0.30 and $0.70 \mathrm{IU} / \mathrm{mL}$ for both reagents [3].

APTT, expressed both as the clotting time (in seconds, sec) and as the patient-to-control ratio, was evaluated using the HemosIL SynthASil reagent (IL). The control clotting time (30.9 sec) was defined as the geometric mean of aPTT measured in the plasma from at least 30 healthy individuals [13]. The aPTT therapeutic range, between 47.0 and $94.0 \mathrm{sec}$, corresponding to patient-to-control ratios in the range from 1.52 to 3.04 , was calculated by plotting aPTT (y axis) vs. anti-Xa activity ( $\mathrm{x}$ axis) evaluated in the plasma of 60 different patients on UFH and was defined to correlate with anti-Xa activity between 0.30 and $0.70 \mathrm{IU} / \mathrm{mL}$ [3]. The within-run precision of the technique, evaluated as the CV using lyophilized plasma samples, was in the range from $1.2 \%$ (for a normal control plasma with a mean $\mathrm{aPTT}=30.3 \mathrm{sec}$ ) to $0.9 \%$ (for a pathological control plasma with a mean $\mathrm{aPTT}=49.3 \mathrm{sec}$ ) whereas the total $\mathrm{CV}$ was in the range from $1.6 \%$ to $2.1 \%$ respectively, as mentioned in the reagent package insert (version 06/2017). The locally defined imprecision of the technique was between $3 \%$ for test results within the normal range and $7 \%$ for aPTTs close to $60 \mathrm{sec}$

Single lots of these reagents were used throughout the study. All assays were automated on the ACL TOP 700 CTS (IL).

\section{Statistical Analysis}

As the distributions of the data were not found to be normal, anti-Xa activity and aPTT results were expressed as the median values with their ranges. Accordingly, analytical comparison of test results obtained at $\mathrm{T} 4 \mathrm{~h}$ and $\mathrm{T} 1 \mathrm{~h}$ was performed using the Wilcoxon signed rank test for paired samples, and the relationship between test results was evaluated using the Spearman's coefficient of rank correlation (rho). P values below 0.05 were considered to be significant. The mean bias (with 95\% confidence interval, $\mathrm{Cl}$ ) between test results obtained at $\mathrm{T} 4 \mathrm{~h}$ and $\mathrm{T} 1 \mathrm{~h}$ were evaluated according to Bland-Altman [14]. The calculated mean bias for aPTT was compared to the desirable 
bias derived from biologic variation (2.3\%) that were published by Ricos C, et al. [15] and updated in electronic version [16], and with the recommended $\left(90^{\text {th }}\right.$ percentile $\mathrm{Cl}$ ) limits of variations for hemostasis tests (18.6\% for aPTTs between 33 and 50 sec., $14.6 \%$ for aPTTs between 50 and 70 sec. and $12.6 \%$ for aPTTs above 70 sec.) published online by the GFHT [17]. The relationship between test results was tested according to Passing-Bablock. The concordance of test results as to whether test results were found within or outside the therapeutic range when measured at T4h and at T1h was evaluated using the kappa score. Usually, reliability was excellent when kappa scores were above 0.8 , good when kappa scores were between 0.6 and 0.8 , moderate when kappa scores were between 0.4 and 0.6 , and poor when kappa scores were below 0.4 [18]. Statistical analysis was performed using the MedCalc software (v.18.11.6, MedCalc Software bvba, Ostend, Belgium). 


\section{Results}

\section{Anti-Xa activity}

Analytical comparison of anti-Xa activity measured after a 4h-storage (T4h) vs. T1h demonstrated a significant $(p<0.0001)$ decrease from a median value of $0.40 \mathrm{IU} / \mathrm{ml}$ (range: $0.10-0.99)$ to 0.35 $\mathrm{IU} / \mathrm{mL}$ (range: $0.11-0.97$ ) (Table 1), even though test results were found to be highly correlated $(\mathrm{r}=+0.961)$. The relationship between test results obtained at $\mathrm{T} 4 \mathrm{~h}$ and $\mathrm{T} 1 \mathrm{~h}$, performed according to Passing and Bablock, was reported in the Figure 1 (panel A). Comparison of test results obtained at $\mathrm{T} 4 \mathrm{~h}$ and $\mathrm{T} 1 \mathrm{~h}$, evaluated according to Bland and Altman, demonstrated a mean bias of -0.05 $\mathrm{IU} / \mathrm{mL}(95 \% \mathrm{Cl}=-0.13+0.03)$ (Figure 2, panel A) corresponding to a mean decrease of $11.9 \%$ $(95 \% \mathrm{Cl}=-36.1+11.3)$ in the measured anti-Xa activity. The mean bias (in $\mathrm{IU} / \mathrm{mL}$ ) was identical for anti-Xa activities below $(n=91,74 \%)$ or above $0.50 \mathrm{IU} / \mathrm{mL}(\mathrm{n}=32,26 \%)$. Such a value was below the imprecision of the technique for values around $0.50 \mathrm{IU} / \mathrm{mL}(16 \%)$, even though it exceeded the between-run CV of the technique (see Patients, Materials and Methods part). Changes were of the same order of magnitude when we analyzed only the first sample collected from each of the 56 patients on UFH (data not shown). If considering 0.30 and $0.70 \mathrm{IU} / \mathrm{mL}$ as the therapeutic range for anti-Xa activity, 12 cases (9.8\%) of discrepancy as whether a test result was subtherapeutical, therapeutical or supratherapeutical were demonstrated. Most of them, corresponding to anti-Xa activities close to the lower limit of the therapeutic range, would not have induced any change in the management of anticoagulation therapy in most of these patients but in two cases (Table 2). The concordance as whether anti-Xa activity was found within or outside the therapeutic range when evaluated at $\mathrm{T} 4 \mathrm{~h}$ and at $\mathrm{T} 1 \mathrm{~h}$ was found to be excellent with a kappa score of 0.813 $(95 \% \mathrm{Cl}=0.7120 .914)$.

In addition, we evaluated the impact of delayed measurement of anti-Xa activity in a subset of 67 tubes that were centrifuged within one hour after collection and were stored capped without separation of the plasma in the vertical position at room temperature until $4 \mathrm{~h}$. Analytical comparison of anti-Xa activity measured in the same tubes demonstrated a significant $(p<0.0001)$ decrease after a $4 \mathrm{~h}$-storage vs. T1h from a median value of $0.44 \mathrm{IU} / \mathrm{ml}$ (range: $0.10-0.89$ ) to 0.42 
$\mathrm{IU} / \mathrm{mL}$ (range: $0.08-0.86)$, with a mean bias of $-0.02 \mathrm{IU} / \mathrm{mL}(95 \% \mathrm{Cl}=-0.06+0.03)$, whereas test result in the paired tubes left unspun for $4 \mathrm{~h}(\mathrm{~T} 4 \mathrm{~h})$ before being processed at the same time was $0.39 \mathrm{IU} / \mathrm{mL}$ (range: $0.09-0.86)$.

To confirm these results, the anti-Xa activity was evaluated using another chromogenic assay (HemosIL Liquid Anti-Xa), in a subset of 62 paired of tubes. Anti-Xa activity was significantly $(p<0.0001$ ) lower at T4h than at T1h: $0.32 \mathrm{IU} / \mathrm{mL}$ (range: $0.10-0.99)$ vs. $0.37 \mathrm{IU} / \mathrm{mL}$ (range: $0.11-$ 0.92), test results being highly correlated $(r=+0.929)$. When compared according to Bland and Altman, test results evaluated at T4h vs. T1h showed a mean negative bias of $-0.04 \mathrm{IU} / \mathrm{mL}$ $(95 \% \mathrm{Cl}=-0.16+0.08)$, identical for activities below $(\mathrm{n}=45,75.8 \%)$ and above $0.50 \mathrm{IU} / \mathrm{Ml}(\mathrm{n}=17$, $24.2 \%)$. This corresponded to a mean decrease of $10.7 \%(95 \% \mathrm{Cl}=-46.2+24.8)$ in the measured anti-Xa activity using that assay. 8 cases of discordant test results (12.9\%) were observed, most of them being found around the lower limit of the therapeutic range, without impact on the management of the anticoagulant therapy. The kappa score was found to be $0.750(95 \% \mathrm{Cl}=0.596$ 0.904).

\section{aPTT}

APTT was significantly shortened $(p<0.0001)$ after a 4 h-storage at room temperature vs. T1h: 46.6 sec. $(27.8$ - 138.0) vs. 54.5 sec. $(31.5$ - 147.3) as shown in the Table 1, even though test results were found to be highly correlated $(r=+0,927)$. The relationship between test results obtained at T4h and T1h, performed according to Passing and Bablock, was reported in the Figure 1 (panel B). The mean bias between test results obtained at T4h and T1h, evaluated according to Bland and Altman, was $-7.9 \mathrm{sec}(95 \% \mathrm{Cl}=-23.3+7.5)$, as shown in Figure 2 (panel B). Such a bias corresponded to a mean decrease in aPTT ratio of $-0.25(95 \mathrm{Cl} \%=-0.75+0.24)$ in absolute value, the relative change being of $-12.1 \%(95 \mathrm{Cl} \%=-34.8+10.6)$. That mean bias far exceeded the imprecision of the technique (between 3\% for normal test results to $7 \%$ for aPTTs close to 60 sec., see Patients Materials and Methods part) and the desirable value of $2.3 \%$ recommended by Ricos et al. $[15,16]$, but remained within the recommended $\left(90^{\text {th }}\right.$ percentile $\left.\mathrm{Cl}\right)$ limits of variation for the bias recommended by the GFHT [17]). 
If considering the aPTT therapeutic range between 47 and $94 \mathrm{sec}$. (or 1.52 and 3.04 when expressed as the patient-to-control ratio) that corresponded to anti-Xa activity between 0.30 and $0.70 \mathrm{IU} / \mathrm{ml}, 29$ cases of discordant test results (23.6\%) were observed. Even though most of them were found around the lower and upper limits of the therapeutic range, such discrepant test results would have induced changes in the management of anticoagulation of 10 patients (Table 2). The concordance as whether aPTT results were found within or outside the therapeutic range when evaluated at T4h and T1h was good, with a kappa score of $0.661(95 \% \mathrm{Cl}=0.5560 .766)$.

The impact of delayed measurement of aPTT in plasma was evaluated in the subset of 67 tubes that were centrifuged within one hour after collection and were stored capped without separation of the plasma in the vertical position at room temperature until $4 \mathrm{~h}$. Analytical comparison of aPTT measured in the same tubes demonstrated a significant $(p=0.0002)$ decrease after a 4h-storage vs. T1h from a median value of $56.6 \mathrm{sec}$ (range: 31.5 - 141.1) to $53.0 \mathrm{sec}$ (range: 29.1 - 134.2), with a mean bias of $-3.5 \sec (95 \% \mathrm{Cl}=-8.9+2.0)$, whereas aPTT test result in the paired tubes left unspun for $4 \mathrm{~h}(\mathrm{~T} 4 \mathrm{~h})$ before being processed at the same time was $48.9 \mathrm{sec}$ (range: 28.5 - 139.5). 


\section{Discussion}

Most current guidelines recommend performing tests aimed at monitoring UFH therapy as soon as possible after blood sampling, usually between $1 \mathrm{~h}$ and $2 \mathrm{~h}$ when blood is collected into citrated tubes $[8,9]$. That delay is far shorter than the usually recommended 4 hour-delay for most coagulation tests [8]. The rationale behind such a short delay relies on the potential for in vitro neutralization of UFH present in the patient's blood by PF4 released from platelet $\alpha$-granules after their activation during or after venipuncture [7]. PF4 is a chemokine of CXC subfamily that is stored in platelet $\alpha$-granules and is released in the plasma after platelet activation [19]. Beside its main patho-physiological role in inflammation process by enhancing chemotaxis, release of histamine by monocytes, adhesion of eosinophils, abrogation of monocytes, apoptosis, and differentiation of monocytes into macrophages [19], released PF4 is able to bind with high affinity heparin, other polysaccharides as well as negatively charged compounds and so to neutralize their biological effects $[7,20,21]$ that could lead to underestimate plasma heparin levels.

To minimize that potential impact, the CLSI Guideline H21-A5 [8] recommends that testing for antiXa activity or aPTT prescribed for monitoring UFH therapy has to be performed within 1 hour after sampling when blood is collected into citrated tubes. A similar recommendation was made by the GFHT for unspun citrated tubes, even though a 2 hour-delay between sampling and processing for unspun tubes, and a 4 hour-delay for collection tubes that were centrifuged within 1 hour were found to be acceptable [9]. However, evidences to support such recommendations are limited. Adcock et al. [22] in their seminal study, evaluated potential changes in aPTT test results after different storage times of either spun or unspun citrated tubes collected from patients on UFH. They concluded to a significant shortening effect of $-15.3 \%$ (in relative value) starting after a $4 \mathrm{~h}$ storage of unspun citrated tubes $(n=8)$ compared to immediate determination $(<1 \mathrm{~h})$, whereas changes were not significant for centrifuged tubes $(n=14)(22)$. In the second part of their study they evaluated at shorter time intervals samples obtained from 3 patients on UFH and found a significant decrease in both anti-Xa activity and aPTT beginning for delays above 1 hour that correlated with an increase in plasma PF4 levels [22]. In a study evaluating samples collected from 
44 patients on UFH, Ray M. [23] reported statistically significant decreases (around $3 \%$ in relative value) in aPTT and anti-Xa activity but without clinical relevance after a 100 min- vs. a 30 minstorage of unspun citrated tubes. In the other hand, two studies reported significant prolongations in aPTT results after prolonged storage of unspun citrated tubes i.e. a $+15.4 \%$ prolongation (in relative value) after an 8 h-storage for Heil et al. [24] and $+11.6 \%$ prolongation after a 6 h-storage for Awad et al. [25].

The maximum delay between blood collection and analysis is indeed a critical point that must be precisely defined for each parameter to avoid sample deterioration that may invalidate analysis results. Actually, errors within the pre-analytical phase far exceed those occurring during the analytical and post-analytical phases, and account for around two third of the total errors recorded in medical laboratories (26), particularly for hemostasis tests [27,28]. Accordingly, accreditation bodies are increasingly requiring laboratories to take responsibility for the extra-analytical phase [29]. So, detailed information about pre-analytical conditions, particularly the delay between specimen collection, its delivery to the laboratory and its processing, has to be provided, according to the College of American Pathologists (CAP) checklist [30] and the International Organization for Standardization (ISO) 15189:2012 norm [31]. As the consequence of the economic pressure that led to a consolidation of clinical laboratories into so-called "core labs" in the last years, the delay between specimen collection and its delivery to the production site has dramatically increase due to longer distances between these two sites. In such conditions a 1 hour-delay should be a limitation, making these recommendations difficult to apply in routine practice. So, the potential impact of longer storage times needed to be further investigated. We here reported a decrease in anti-Xa activity when evaluated after a 4h-storage vs. a 1h-delay that had limited impact in the management of UFH therapy, even though it was statistically significant. Such a decrease was demonstrated both in unspun tubes and in centrifuged tubes kept capped without separation of the plasma at room temperature for $4 \mathrm{~h}$. However, the latter demonstrated smaller changes with a lower bias between test results $(-0.02 \mathrm{IU} / \mathrm{mL}$ vs. $-0.05 \mathrm{IU} / \mathrm{mL})$ and a lower variability in the changes. Accordingly, the present results suggest that it seems to be acceptable extending to 4 hours at room temperature the delay between blood collection and measurement of anti-Xa activity 
prescribed for monitoring UFH treatments when blood is collected into citrated tubes, at least when evaluated using the two tested reagents. Of note, getting heparin dosing consistently into the therapeutic range, especially during initiation of anticoagulation in high-risk patients such as those who have suffered a recent stroke, is very challenging and lag in receiving results would surely further compromise quality of anticoagulation control. So, the recommendation is to process these samples as soon as possible, so that the clinical ward can adapt UFH dosage according to the results of biological tests such as anti-Xa activity. Nevertheless, our results suggest that a $4 \mathrm{~h}-$ delay would be allowed for samples that need to be shipped off site for analysis. In such a case, if compatible with the patient's state, the prescription of more modern anticoagulants with broader dose-response curves and not requiring laboratory monitoring could be a potential alternative to UFH. In contrast, as changes in aPTT results were found to be of a greater order of magnitude and potentially clinically relevant, recommending a shorter delay i.e. 1-2 hours should be justified. It could be mentioned that in order to circumvent the potential in vitro neutralization of UFH by PF4, an alternative option could be using collection tubes containing CTAD (tri-Na citrate, theophylline, adenosine, and dipyridamole) instead of tri-Na citrate as the anticoagulant solution [32]. CTAD is a mixture of anticoagulant and platelet inhibitors aimed at stopping platelet activation, and consequently the release of PF4. By "stabilizing" anti-Xa and aPTT test results in blood collected from heparinized patients, this would enable monitoring UFH treatments in samples collected up to 4 hours prior $[8,9,33]$. Even though, the limited stability of the CTAD anticoagulant solution, particularly its high sensitivity to light [34], as well as the need for such a specific "nonconventional" and more expensive collection tube precludes generalization of its use in routine coagulation testing.

One potential limitation of the present study could be the inclusion criteria of the studied population. We decided to include patients with recent ischemic stroke and not VTE or ACS patients for logistical reasons as the Neurology Department was close to the laboratory. Even though the coagulation equilibrium could be different in these patients, difference in their response to UFH was very unlikely. In that connection, in a very recently published study (article made available on-line during the review process of our manuscript), Billoir $\mathrm{P}$, et al. evaluated the 
stability, of anti-Xa activity and aPTT, evaluated using other reagents, in uncentrifuged tubes collected from acute patients from medical and cardiac surgery ICUs of a French ternary hospital, who were treated with full dose UFH. As the result, after having evaluated the stability of these parameters for up to $8 \mathrm{~h}$, they concluded that anti-Xa could be validly evaluated in uncentrifuged citrated tubes collected $4 \mathrm{~h}$ prior [35].

In conclusion, the results of the present study suggest that the delay between blood collection and measurement of anti-Xa activity prescribed for monitoring UFH treatments could be safely extended to $4 \mathrm{~h}$. As changes in aPTT were more clinically relevant, the maximum delay should remain around $1-2 \mathrm{~h}$. These finding could provide another clue, beside the better standardization, to promote the use of anti-Xa activity instead of aPTT for monitoring UFH therapy. 


\section{References}

1. Damus PS, Hicks M, Rosenberg RD. Anticoagulant action of heparin. Nature 1973; 246: 355-7.

2. Rosenberg RD. Biochemistry of heparin antithrombin interactions, and the physiologic role of this natural anticoagulant mechanism. Am J Med 1989; 87 (3B): 2S-9S.

3. Garcia DA, Baglin TP, Weitz JI, Samama MM; American College of Chest Physicians. Parenteral anticoagulants: Antithrombotic therapy and prevention of thrombosis, 9th ed: American College of Chest Physicians Evidence-Based Clinical Practice Guidelines. Chest 2012; 141 (2 Suppl): e24S-43S.

4. Anand SS, Yusuf S, Pogue J, Ginsberg JS, Hirsh J. Organization to Assess Strategies for Ischemic Syndromes Investigators. Relationship of activated partial thromboplastin time to coronary events and bleeding in patients with acute coronary syndromes who receive heparin. Circulation 2003; 107: 2884-8.

5. Francis JL, Groce JB 3rd; Heparin Consensus Group. Challenges in variation and responsiveness of unfractionated heparin. Pharmacotherapy 2004; 24 (8 Pt 2): 108S-19S.

6. Levine MN, Hirsh J, Gent M, Turpie AG, Cruickshank M, Weitz J, Anderson D, Johnson M. A randomized trial comparing activated thromboplastin time with heparin assay in patients with acute venous thromboembolism requiring large daily doses of heparin. Arch Intern Med 1994; 154: 4956.

7. Denton J, Lane DA, Thunberg L, Slater AM, Lindahl U. Binding of platelet factor 4 to heparin oligosaccharides. Biochem J 1983; 209: 455-60.

8. Adcock DM, Hoefner DM, Kottke-Marchant K, Marlar RA, Szarmozi DI, Wuranek DJ. Collection, transport, and processing of blood specimen for testing plasma-based coagulation assays and molecular hemostasis assays; Approved Guideline-Fifth Edition. CLSI Document H21-A5. Clinical and Laboratory Standards Institute, Wayne, PA, USA, 2008; Vol. 28: n 5, pp.1-33.

9. Groupe Francophone d'Etude sur l'Hémostase et la Thrombose (GFHT). http://site.geht.org/recommandations-pre-analytiques-hemostase-specialisee/ 
10. WHO. Guidelines for thromboplastin and plasma used to control oral anticoagulant therapy. WHO Technical Report Series. 1999; 889: 64-93.

11. Walker ID. Blood collection and sample preparation: pre-analytical variation. In: Jespersen J, Bertina RM, Haverkate F, eds. Laboratory techniques in thrombosis - A manual. 2nd revised edition of ECAT assay procedures. Kluwer Academic Publishers, Dordrecht, The Netherlands, 1999; pp 21-28.

12. Polack B, Schved J-F, Boneu B, on behalf of the 'Groupe d'Etude sur l'Hémostase et la Thrombose' [GEHT]. Preanalytical recommendations of the 'Groupe d'Etude sur l'Hémostase et la Thrombose' (GEHT) for venous blood testing in hemostasis laboratories. Haemostasis 2001; 31 : 61-8.

13. Marlar RD, Cook J, Johnston M, Kitchen S, Machin SJ, Shafer D, Worfolk R. One-Stage Prothrombin Time (PT) Test and Activated Partial Thromboplastin Time (APTT) Test, 2nd Edition CLSI Document H47-A2. Clinical and Laboratory Standards Institute, Wayne, PA, USA, 2008; Vol. 28: $n^{\circ} 20, p p .1-32$.

14. Bland JM, Altman DG. Statistical methods for assessing agreement between two methods of clinical measurement. Lancet 1986; I: 307-10.

15. Ricós C, Alvarez V, Cava F, García-Lario JV, Hernández A, Jiménez CV, Minchinela J, Perich C, Simón M. Current databases on biological variation: pros, cons and progress. Scand J Clin Lab Invest 1999; 59: 491-500.

16. Ricós C, Alvarez V, Cava F, García-Lario JV, Hernández A, Jiménez CV, Minchinela J, Perich C, Simón M. Desirable specifications for total error, imprecision, and bias, derived from intra- and inter-individual biologic variation. https://www.westgard.com/biodatabase1.htm\#albumin

17. Normes d'acceptabilité en hémostase. Propositions du groupe de travail du GEHT. http://site.geht.org/accreditation-hemostase-pre-analytique/

18. Cohen J. Weighted kappa: Nominal scale agreement with provision for scaled disagreement or partial credit. Psychological Bulletin, 1968; 70: 213-20.

19. Klinger MHF. Inflammation. In Platelets, AD Michelson Editor. Academic Press, San Diego, CA, 2002: pp 459-67. 
20. Munoz EM, Linhardt RJ. Heparin-binding domains in vascular biology. Arterioscler Thromb Vasc Biol 2004; 24: 1549-57.

21. Levine SP, Sorenson RR, Harris MA, Knieriem LK. The effect of platelet factor 4 (PF4) on assays of plasma heparin. $\mathrm{Br} \mathrm{J}$ Haematol 1984; 57: 585-96.

22. Adcock D, Kressin D, Marlar RA. The effect of time and temperature variables on routine coagulation tests. Blood Coagul Fibrinolysis 1998; 9: 463-70.

23. Ray M. Stability of the activated partial thromboplastin time used to monitor unfractionated heparin. J Thromb Haemost 2008; 6: 1817-9.

24. Heil W, Grunewald R, Amend M, Heins M. Influence of time and temperature on coagulation analytes in stored plasma. Clin Chem Lab Med 1998; 36: 459-62.

25. Awad MA, Eldeen OA, Ibrahim HA. Stability of activated partial thromboplastin time (APTT) test under different storage conditions. Hematology 2006; 11: 311-5.

26. Plebani M. The detection and prevention of errors in laboratory medicine. Ann Clin Biochem 2010; 47(Pt 2): 101-10.

27. Salinas M, López-Garrigós M, Yago M, Ortuño M, Carratala A, Aguado C, Díaz J, RodriguezBorja E, Chinchilla V, Esteban A, Laíz B, Lorente MÁ, Uris J. Quality assessment for preanalytical phase in clinical laboratory: a multicentric study. Rev Calid Asist 2011; 26: 264-8.

28. Favaloro EJ, (Adcock) Funk DM, Lippi G. Pre-analytical variables in coagulation testing associated with diagnostic errors in hemostasis. Laboratory Medicine 2012; 43: 1-10.

29. Hawkins R. Managing the pre- and post-analytical phases of the total testing process. Ann Lab Med 2012; 32: 5-16.

30. College of American Pathologists. Laboratory accreditation program. Laboratory general checklist. College of American Pathologists. Northfield, IL, USA, 2010.

31. International Organization for Standardization. ISO 15189: Medical laboratories - particular requirements for quality and competence. Revision 2012. International Organization for Standardization, Geneva, Switzerland, 2012. 
32. Contant G, Gouault-Heilmann M, Martinoli JL. Heparin inactivation during blood storage: its prevention by blood collection in citric acid, theophylline, adenosine, dipyridamole-C.T.A.D. mixture. Thromb Res 1983; 31: 365-74.

33. van den Besselaar AMHP, Meeuwisse-Braun J, Jansen-Grüter R, Bertina RM. Monitoring heparin therapy by the activated partial thromboplastin time - The effect of preanalytical conditions. Thromb Haemost 1987; 57: 226-31.

34. Polack B, Barro C, Mossuz P, Pernod G. Inadequate quality of a blood collection tube containing an anticoagulant/platelet inhibitor mixture. Thromb Haemost 1997; 77: 1035-6.

35. Billoir P, Clavier T, Guilbert A, Barbay V, Chrétien MH, Fresel M, Abriou C, Girault C, Le Cam Duchez V. Is citrate theophylline adenosine dipyridamole (CTAD) better than citrate to survey unfractionated heparin treatment? Has delayed centrifugation a real impact on this survey? J Thromb Thrombolysis. 2019 Aug;48(2):277-283. doi: 10.1007/s11239-019-01882-1. 


\begin{tabular}{|c|c|c|c|c|}
\hline & T1h & T4h & $\mathrm{p}^{*}$ & Bias $^{* *}(95 \% \mathrm{Cl})$ \\
\hline Anti-Xa Hyphen $(\mathrm{IU} / \mathrm{mL})$ & $0.40(0.10-0.99)$ & $0.35(0.11-0.97)$ & $<0.0001$ & $-0.05(-0.13+0.03)$ \\
\hline aPTT SynthASil (sec) & $\begin{array}{c}54.5(31.5-147.3) \\
\text { (ratio) }\end{array}$ & $\begin{array}{c}4.76(1.02-4.75) \\
1.50(0.96-4.45)\end{array}$ & $\begin{array}{c}<0.0001 \\
<0.001\end{array}$ & $\begin{array}{c}-7.9(-23.3+7.5) \\
(-0.75+0.24)\end{array}$ \\
\hline
\end{tabular}

Table 1: Anti-Xa activity (in IU/mL) and aPTT (expressed in sec. and as the patient to control ratio) evaluated in 123 plasma samples from patients treated with unfractionated heparin. Measurement was performed within 1 hour after blood collection (T1h) and after a 4 hour-storage (T4h) of unspun collection tubes at room temperature. Test results were expressed as the median values with their ranges.

* Analytical comparison was performed using the Wilcoxon test for paired results; ${ }^{* *}$ Bias (with 95\% confidence interval, Cl) was calculated according to Bland and Altman 


\begin{tabular}{|l|l|l|}
\hline & $\begin{array}{l}\text { Test result within the therapeutic } \\
\text { range at T1h and below it at T4h }\end{array}$ & $\begin{array}{l}\text { Test result above the therapeutic } \\
\text { range at T1h and within it at T4h }\end{array}$ \\
\hline Anti-Xa Hyphen $(\mathrm{IU} / \mathrm{mL})$ & $0.32 / 0.23 ; 0.34 / 0.18 ; 0.34 / 0.28$ & $0.73 / 0.67$ \\
& $0.35 / 0.27 ; 0.35 / 0.28 ; 0.36 / 0.29$ & \\
$\mathrm{n}=12 / 123(9.8 \%)$ & $0.38 / 0.24 ; 0.38 / 0.28 ; 0.44 / 0.29$ & \\
& $0.35 / 0.28 ; 0.37 / 0.26$ & \\
\hline & & \\
aPTT SynthASil (ratio) & $1.53 / 1.09 ; 1.55 / 1.49 ; 1.55 / 1.41$ & $3.06 / 2.50 ; 3.06 / 2.97 ; 3.09 / 2.56$ \\
& $1.56 / 1.25 ; 1.57 / 1.19 ; 1.57 / 1.24$ & $3.13 / 2.86 ; 3.16 / 2.51 ; 3.16 / 2.80$ \\
$\mathrm{n}=29 / 123(23.6 \%)$ & $1.58 / 1.22 ; 1.59 / 1.21 ; 1.59 / 1.42$ & $3.17 / 2.84 ; 3.44 / 2.40 ; 3.16 / 3.01$ \\
& $1.63 / 1.37 ; 1.64 / 1.37 ; 1.70 / 1.49$ & $3.39 / 2.63$ \\
& $1.73 / 1.33 ; 1.76 / 1.38 ; 1.80 / 1.50$ & \\
& $1.83 / 1.43 ; 2.03 / 1.41 ; 2.31 / 1.45$ & \\
& $2.00 / 0.95$ & \\
& & \\
\hline
\end{tabular}

Table 2: Discrepant anti-Xa activity and aPTT test results measured in citrated tubes within 1 hour (T1h) after blood collection and after a 4 hour-storage (T4h) of unspun collection tubes at room temperature before being centrifuged and subsequently analyzed. 


\section{Legends to the Figures}

Figure 1: Relationship according to Passing-Bablock of anti-Xa activity (in IU/mL, panel A) and activated partial thromboplastin time (aPTT, expressed in sec, panel B) evaluated after a 4h- (T4h, $y$ axis) vs. a $<1$ h-storage (T1h, $x$ axis) at room temperature of unspun citrated blood tubes $(n=123)$ collected from patients treated with unfractionated heparin.

Figure 2: Comparison, performed according to Bland-Altman, of anti-Xa activity (in IU/mL, panel A) and activated partial thromboplastin time (aPTT, is sec, panel B) measured after a 4h- (T4h) vs. a $<1 \mathrm{~h}$-storage $(T 1 h)$ at room temperature of unspun citrated blood tubes $(n=123)$ collected from patients treated with unfractionated heparin. Results are plotted as the test results measured at T1h ( $\mathrm{x}$ axis) vs. the differences in anti-Xa or aPTT (y axis). 
A

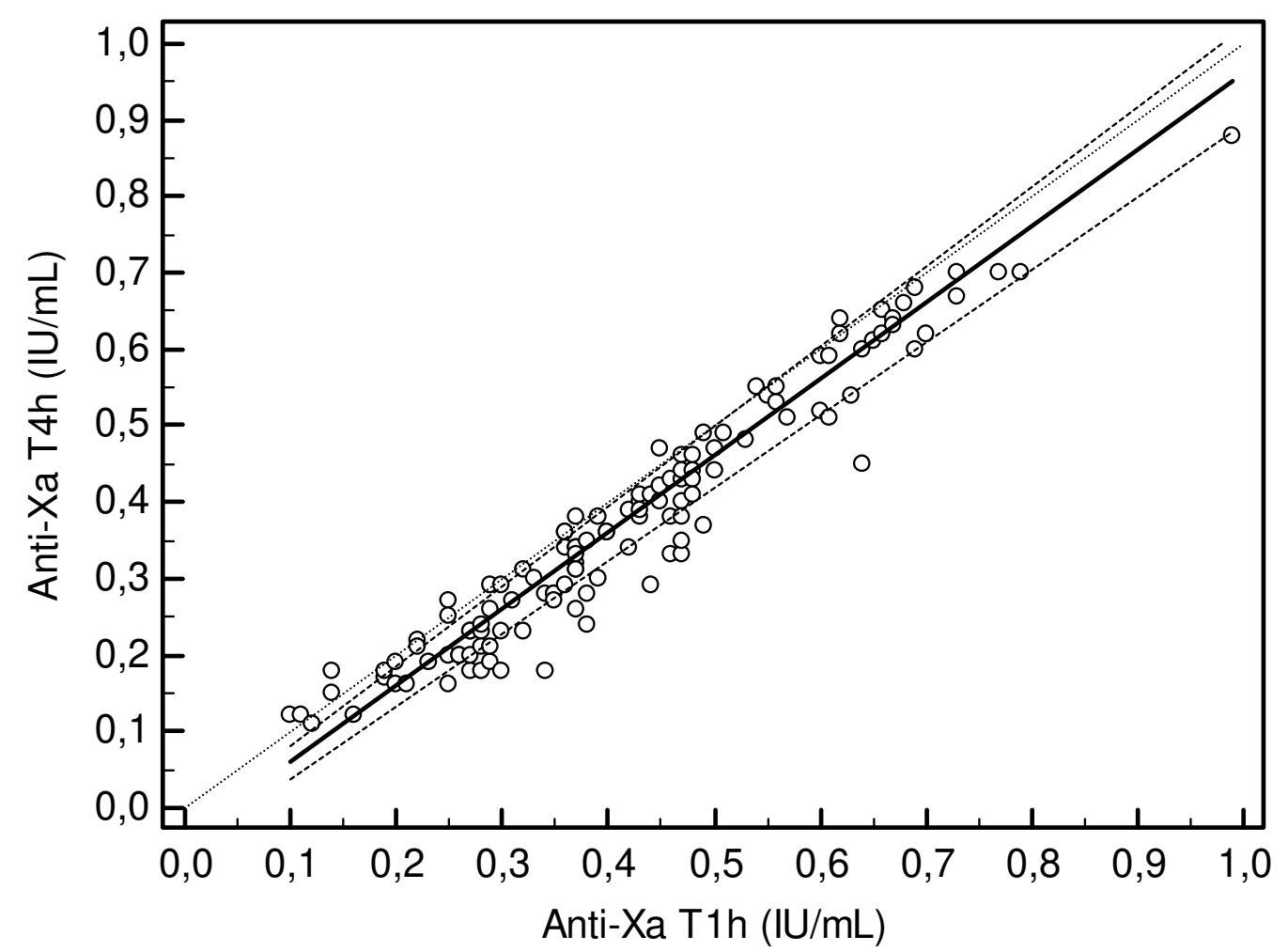

B

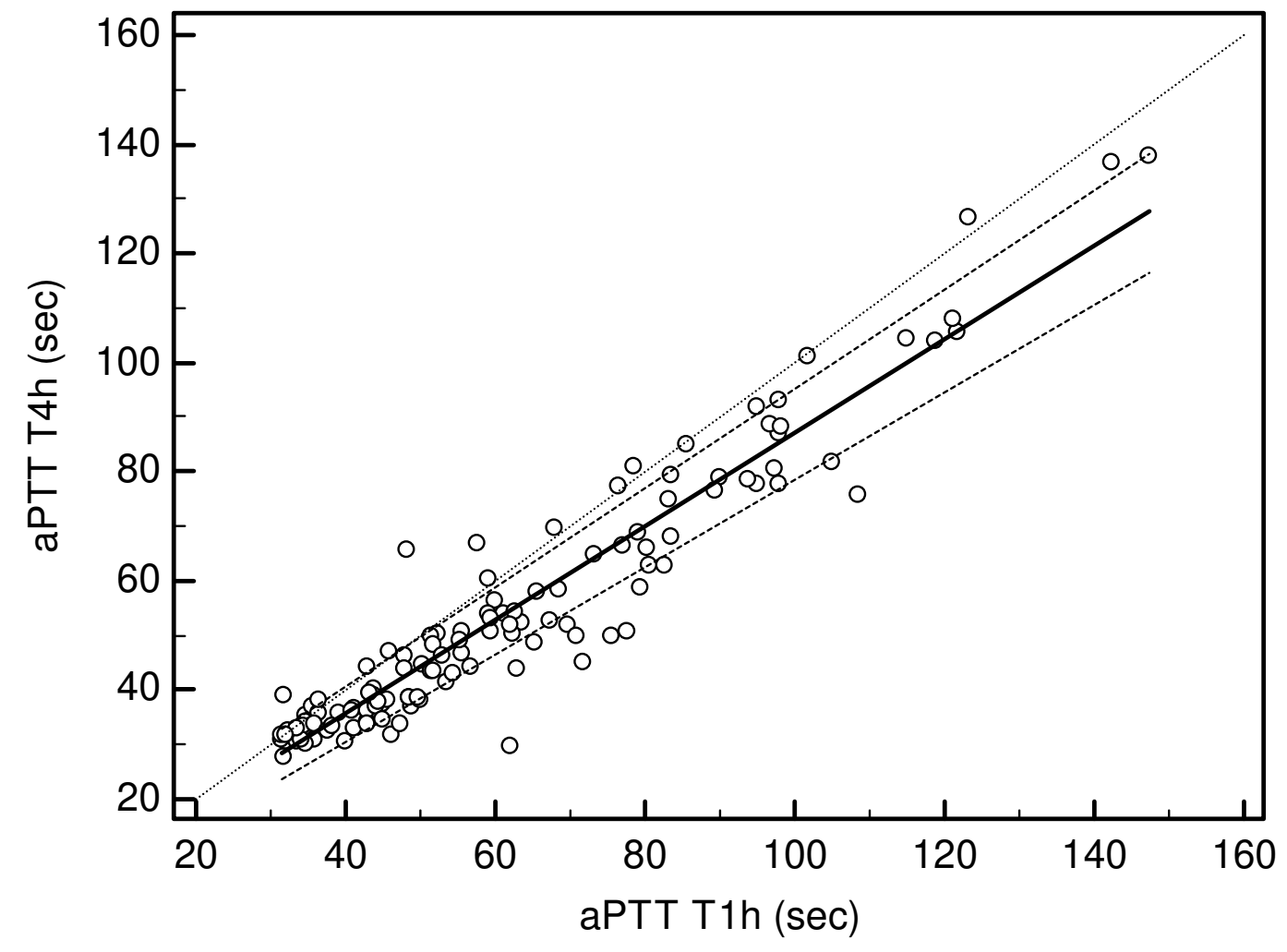




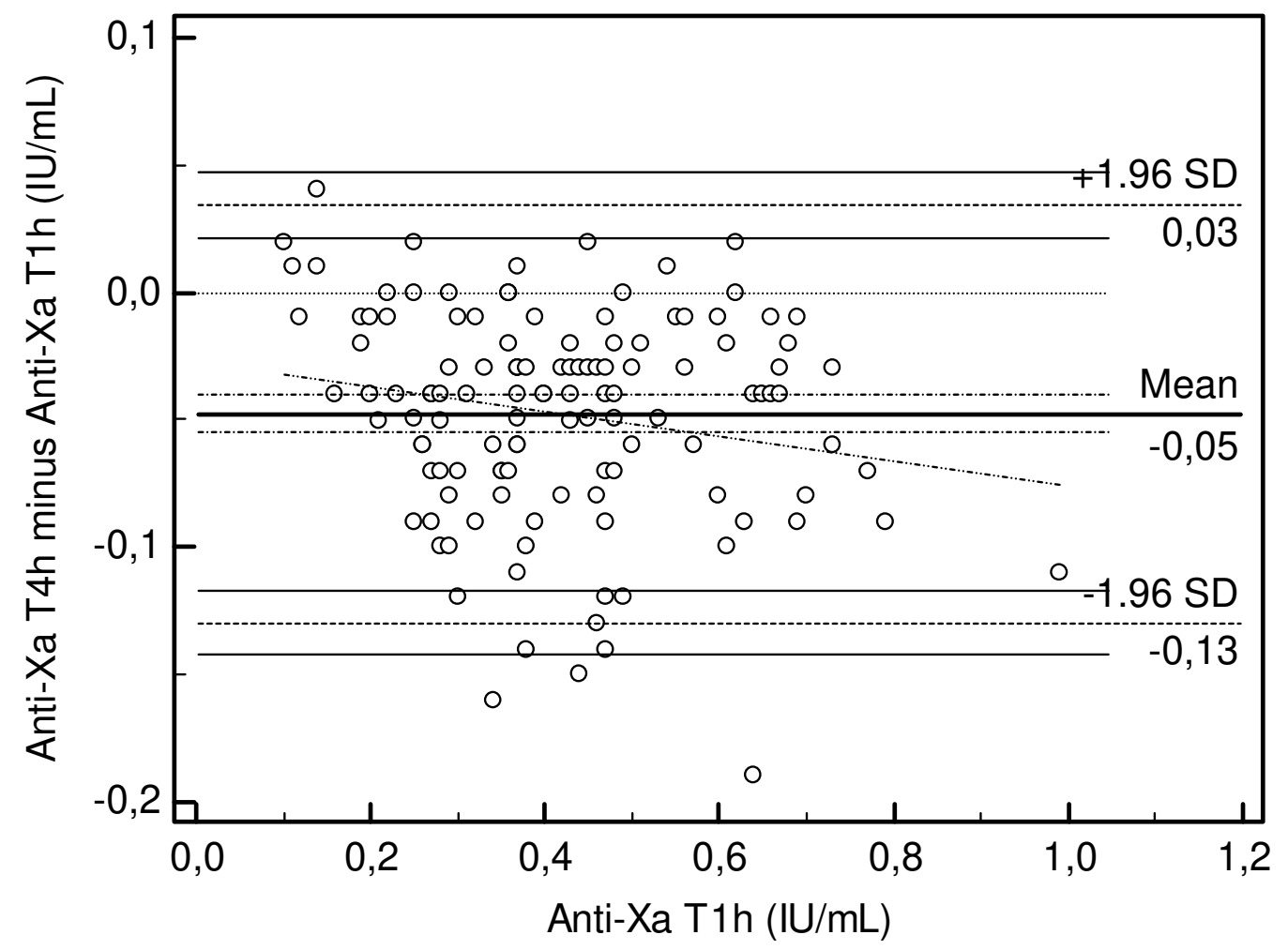

B

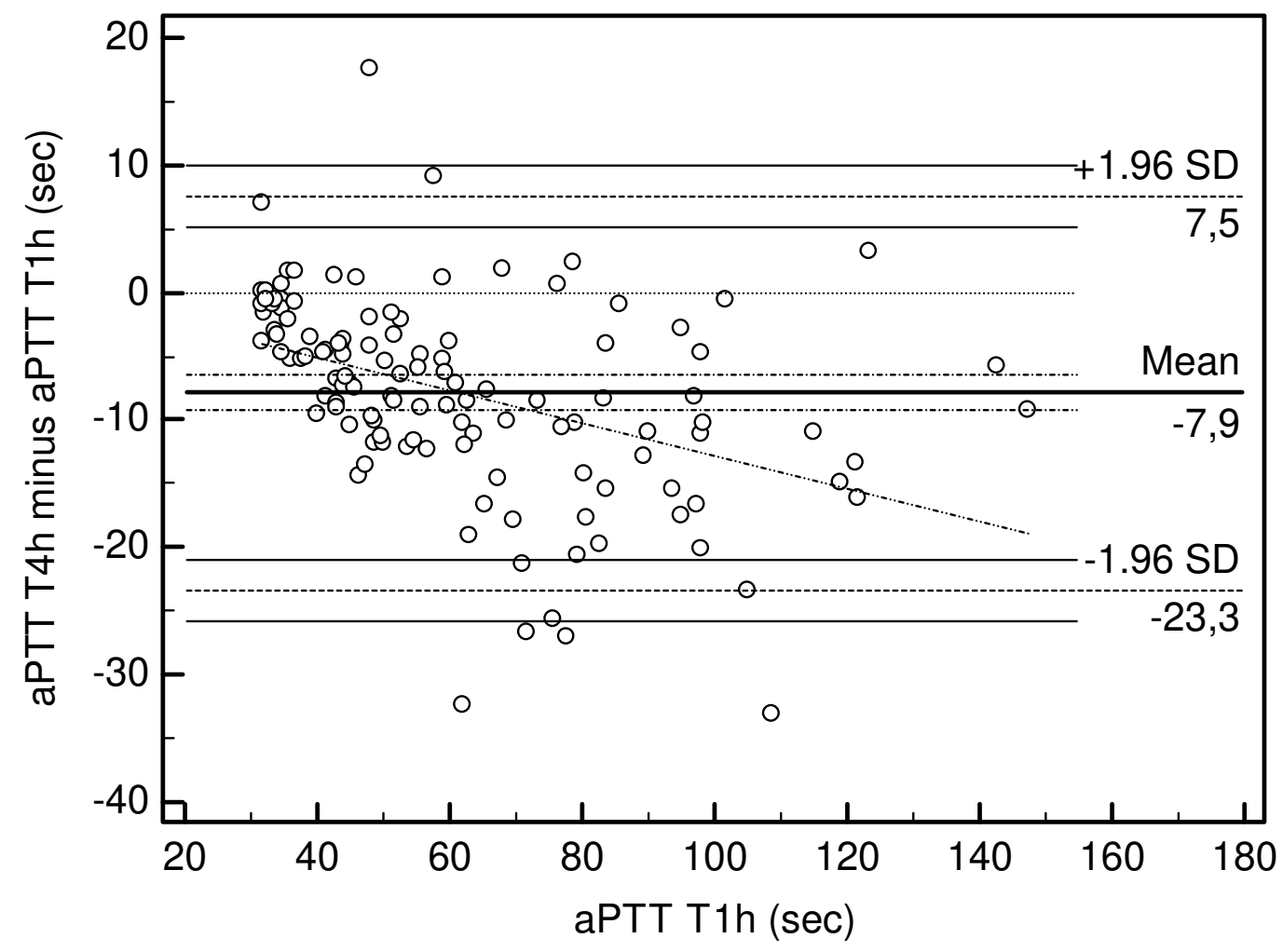

